Journal of Advanced Research in Fluid Mechanics and Thermal Sciences

Journal homepage: www.akademiabaru.com/arfmts.html ISSN: 2289-7879

\title{
Nanotechnology for Molecular Imaging of Atherosclerosis: Current Design and Approaches
}

\author{
Mardhiah Maslizan ${ }^{1}$, Intan Diana Mat Azmi ${ }^{1,2,{ }^{*}}$, Adila Mohamad Jaafar ${ }^{1,2}$, Muhammad Salahuddin \\ Haris $^{3}$ \\ 1 Department of Chemistry, Faculty of Science, Universiti Putra Malaysia, 43400 UPM Serdang, Selangor Darul Ehsan, Malaysia \\ 2 Centre of Foundation Studies for Agricultural Science, Universiti Putra Malaysia, 43400 UPM Serdang, Selangor Darul Ehsan, Malaysia \\ 3 Department of Pharmaceutical Technology, Kulliyyah of Pharmacy, International Islamic University Malaysia, 25200 Kuantan, Pahang Darul \\ Makmur, Malaysia
}

\section{ARTICLE INFO}

\section{Article history:}

Received 18 October 2020 Received in revised form 20 February 2021 Accepted 5 March 2021

Available online 1 April 2021

\section{Keywords:}

Nanotechnology; atherosclerosis; nanoparticles; nanomedicines imaging

\section{ABSTRACT}

\begin{abstract}
Atherosclerosis complications such as myocardial infarction or stroke is still one of the most critical causes of death worldwide. Advance and innovative diagnostic technologies are urgently required to discover an early stage of the disease, such as plaque instability and thrombosis. A combination of molecular imaging probes based on well-designed nanomaterials with leading-edge imaging methods is currently concreting the direction for novel and distinctive approaches to examine the inflammatory growth in atherosclerosis. Over the past several decades, an exceptional understanding of the biological nature of atherosclerosis provides unique opportunities to better treat atherosclerotic disease with targeted imaging and nanomedicines. Consequently, tremendous development has been initiated in the nanotechnology application; the leading engineering tools working at molecular range, which is designed for diagnostic and therapeutic approach, called theranostic. This review underlying ideas involving the potential and development of molecular imaging technologies that had been invented for studying atherosclerosis. We envisage that many molecular imaging methods will become valuable assistants to the clinical management of targeted treatment in the atherosclerosis disease together with their challenges and future perspective in clinical translation.
\end{abstract}

\section{Introduction}

Cardiovascular diseases (CVDs) are the leading causes of death [1]. According to a study by the World Health Organization, the World Heart Federation and the World Stroke Organization stated that from two-thirds of all deaths in the world which belong to non-communicable diseases, almost $50 \%$ are from CVDs alone [2]. Furthermore, the key for overcoming the challenges in the prevention of cardiovascular disease require global solutions that should comprise of economic, political, public health, technological solution, training for health professionals and researchers [2]. Besides, many popular conventional therapeutic CVD treatments, for instance; anti-hyperlipidemic drugs, anti-

\footnotetext{
* Corresponding author.

E-mail address: intandiana@upm.edu.my
}

https://doi.org/10.37934/arfmts.81.2.124138 
inflammatory drugs, anticoagulant drugs and anti-hypertensive drugs, poorly treat the inflammatory processes during plaque formation [4]. As a result, researchers and clinicians continue to explore other innovative therapeutic methods using nanomedicines-based approaches to help fight the disease with better precision and effect [3].

Zdrojewicz et al., [5] reported that the injection of amino acids 2-26 loaded nanoparticles (Col IV-Ac2-26 NPs) that targeted the collagen IV on atherosclerotic mice revealed their potential in treating chronic atherosclerosis by substantially improved the characteristics of the atherosclerotic plaque, for instance, increased the protective collagen layer that further minimized the plaque necrosis [5]. Another supportive finding revealed that phosphatidylserine-containing nanoparticles combined with curcumin could greatly influence the atherosclerotic plaque stability by efficiently blocked lipid build-up, inhibit pro-inflammatory factor production and encouraged the flow of antiinflammatory cytokines via greater transcription and secretion levels of anti-inflammatory factors (TGF- $\beta$ and IL-10) from oxLDL activated macrophages after the uptake of PS-containing curcuminloaded nanostructured lipid carriers(Cur-mNLCs) [6]. Thus, these nanoparticles can be used to transport active constituents together with anti-inflammatory agents, and significantly treat atherosclerotic lesion [5-8].

Nanotechnologies are defined as current approach in designing, classifying and functionalizing models or tools, by modifying the structures and the molecular sizes at nano-meter level [7]. As nanotechnologies advanced into clinical medicine, novel techniques utilizing nanomedicines to treat cardiovascular diseases has been undertaken to resolve the complex pathogenesis of atherosclerosis [8]. Different imaging technologies are being developed to visualize various features of vessel walls that could indicate the onset of acute atherosclerotic cases. For example, Four-dimensional (4D)-flow magnetic resonance imaging (MRI) offers a detailed study of complex blood circulation in the aortic valve and thoracic aorta with short scanning times, enhanced pre-processing study and visualization equipment, thus promoting its clinical practicality [9].

Nanotechnology is a promising method for imaging and therapy of atherosclerosis due to its small size nanoparticles (NPs) [10]. These nanoparticles can be loaded with bio-active compounds such as drugs and contrast agents for targeting, imaging and lesion treatment thereby resulted in accurate diagnosis and treatment of atherosclerosis [11]. The choice of the standard imaging technique, however, varies based on the biological features to be assessed, the readiness of molecular probes, and the procedure's approachability [10]. Table 1 showed several types of nanoparticles that have potential applications for atherosclerosis imaging. Targeted-imaging nanoparticles may also be used to specifically obtain information on the detail development and treatment of the specific lesions [11]. Recently, Schnitzler et al., [12] reported that the use of nanomedicines in drug delivery and tracer imaging improves the sensitivity and effectiveness of these agents intended for atherosclerotic diseases prevention by means of targeting the resident macrophages and activating the endothelial cells. Nanomedicines-based treatment has become an essential strategy for delivering safe and targeted nanoparticles to further reduce the residual risk of cardiovascular events [13]. 
Table 1

Current research development on imaging-based nanoparticles for atherosclerosis

\begin{tabular}{|c|c|c|c|c|c|}
\hline $\begin{array}{l}\text { Nanoparticles and contrast } \\
\text { agent }\end{array}$ & $\begin{array}{l}\text { Size } \\
(\mathrm{nm})\end{array}$ & Imaging & Target & Result & $\begin{array}{l}\text { Year } \\
\text { [Reference] }\end{array}$ \\
\hline $\begin{array}{l}\text { Fluorescein isothiocyanate and } \\
\text { peptide conjugated mesoporous } \\
\text { silicon nanoparticle (FITC-VHP- } \\
\text { Fe3O4@SiO2) }\end{array}$ & 50 & MRI & VCAM-1 & $\begin{array}{l}\text { High affinity on the plaque by } \\
\text { reducing } \mathrm{T} 2 \text { relaxation time for } \\
\text { negative enhancement of } \mathrm{T} 2 \text { - } \\
\text { weighted images. }\end{array}$ & $\begin{array}{l}2019 \\
{[14]}\end{array}$ \\
\hline $\begin{array}{l}\text { Tetrazine conjugated 68Ga iron } \\
\text { oxide nano-radiomaterials } \\
\text { (68Ga-NRM-TZ) }\end{array}$ & 15 & $\begin{array}{l}\text { PET } \\
\text { combined } \\
\text { with MRI }\end{array}$ & IgM antibody & $\begin{array}{l}\text { Selective accumulation in } \\
\text { atherosclerotic plaques, by } \\
\text { clearly providing simultaneous } \\
\text { PET and T1-MRI fluorescence } \\
\text { signals. }\end{array}$ & $\begin{array}{l}2019 \\
{[15]}\end{array}$ \\
\hline $\begin{array}{l}\text { Superparamagnetic iron-oxide } \\
\text { nanoparticles conjugated with } \\
\text { the fusion-protein (EGFP-EGF1- } \\
\text { SPIONs) }\end{array}$ & $\begin{array}{l}9.66 \pm \\
1.05\end{array}$ & $\mathrm{MRI}$ & Tissue factor & $\begin{array}{l}\text { Good integrity and stability via } \\
\text { reduced focal negative signals } \\
\text { of T2-weighted images }\end{array}$ & $\begin{array}{l}2019 \\
{[16]}\end{array}$ \\
\hline $\begin{array}{l}\text { Curcumin incorporated titanium } \\
\text { dioxide nanoparticles (CTNPs) } \\
\text { conjugated MCP-1 antibody }\end{array}$ & 29.5 & $\mathrm{MRI}$ & Aortic region & $\begin{array}{l}\text { Improved in vivo contrast at } \\
\text { aortic regions by reducing } \\
\text { longitudinal (T1) and transverse } \\
\text { (T2) relaxation times of the } \\
\text { water protons. }\end{array}$ & $\begin{array}{l}2020 \\
{[17]}\end{array}$ \\
\hline
\end{tabular}

*T1 \& T2 refer to the relaxation time constant in MRI; where the T1 provides positive contrast effect (gadolinium particles), while $\mathrm{T} 2$ provides negative contrast effect (iron oxide particles). The contrast variances between T1 and T2 may enable the plaque segmentation in different constituents.

\section{The Emergence of Nanoparticles for Diagnosis and Therapy of Atherosclerosis}

Among many of the imaging techniques, high-resolution magnetic resonance imaging (MRI) has started to emerge in the ' 90 s as a valuable imaging modality that offered high soft tissue contrast for characterization of atherosclerotic arteries in a noninvasive and nondestructive way. Taking advantage on the tools, Weissleder et al., [18] developed a promising contrast agent consisted of an ultrasmall superparamagnetic iron oxide (USPIO) that are about $10 \mathrm{~nm}$ in particle size that may potentially use as an intravenous contrast agent for the lymphatic system and other organs such as in brain and bone marrow for MRI. The work also reported on the comparison between dextrancoated ultra-small superparamagnetic iron oxide (USPIO) nanoparticles with the superparamagnetic iron-oxide (AMI-25) nanoparticles. The T1 effect of dextran-coated USPIO in rats showed a considerably longer blood half-life than traditional AMI-25, suggesting that the dextran-coated USPIO particles is not immediately detected, opsonized, and phagocytosed by mononuclear phagocytic system (MPS) of the liver and spleen [18]. In addition, the small size and plasma half-life prolongation of the USPIO allows this agent to reach the capillary wall and have a more widespread distribution of tissue, including lymph node and bone marrow uptake by the MPS than the AMI-25 [18]. Thus, utilization of dextran was a good strategy to improve the uptake and the specificity for macrophages in atherosclerotic plaques [18]. Moreover, USPIO was also been applied in a magnetic resonance angiography (MRA) because of the T1 shortening effect and their long intravascular half-life, thus increasing vascular signal intensity and improving the visualization of arteries and veins from the thoracic region [19]. Ruehm et al., [20] investigated the use of USPIO nanoparticles as a marker for atherosclerosis-associated inflammatory changes in the vessel wall in hyperlipidemic rabbits. As a result, USPIOs were phagocytosed by macrophages in a sufficient quantity to cause iron-induced susceptibility effects within the atherosclerotic plaques of hyperlipidemic rabbits. Besides USPIO, a lipid-encapsulated perfluorocarbon emulsion with gadolinium targeted on fibrin, and a 
peptidomimetic vitronectin antagonist ligand was applied. The studies revealed a dense accumulation of nanoparticles on the targeted sites of fibrin clots $[21,22]$. These results suggested the potential for sensitive and specific detection of arterial thrombi and the progression of the unstable atherosclerotic plaque [21,22].

Another promising strategy for MRI is the application of annexin A5-functionalized nanoparticle of phosphatidylserine (PS) exposing cells in atherosclerotic lesions was developed [23]. T1-weighted MRI revealed enhanced uptake of the annexin A5-functionalized nanoparticles by PS-expressing cells within atherosclerotic mice lesions in the abdominal aorta [23]. Thus, the annexin A5-conjugated functionalized nanoparticles displayed the capabilities to assess cell types that are considered to contribute to plaque instability and in the evaluation of atherosclerotic lesion phenotype [23]. Wang et al., [24] continued the groundwork of nanoparticles emergence by developing ultralow gadolinium-manganese nanoparticles -(MnOL-Gd NP) for MRI on av $\beta 3$-integrin. This $\mathrm{MRI}$ agent provided efficient in vivo detection of atherosclerotic angiogenesis, with most of the contrast localized within the plaque and media of the diseased aorta [24]. Importantly, this MR molecular imaging agent was readily bio-eliminated via the biliary system with no sign of toxicity [24]. Collectively, $\alpha \mathrm{v} \beta 3-\mathrm{MnOL}-\mathrm{Gd}$ NP offered a practical, safer vehicle for neovascular imaging in atherosclerosis [24].

Besides extensive research on $\mathrm{MRI}$, other imaging techniques such as computed tomography (CT), fluorescence and photoacoustic imaging with or without combination with MRI techniques (e.g., MRI-fluorescence) were also developed for diagnosis and/or mapping of atherosclerosis (Figure 1). For instance, Hyafil et al., [25] developed an iodinated nanoparticulate contrast agent (for instance, N1177) for computed tomography (CT) imaging. A substantial signal enhancement was detected in the plaques after the injection of the iodinated nanoparticulate contrast agent, which was not possible with the iopamidol [25]. Thereby, the N1177 may become an excellent contrast agent for the evaluation of coronary arteries via CT imaging [25]. In the year of 2011, Uchida et al., [26] developed a protein-cage nanoparticle that possesses a macrophage-targeting peptide (LyP-1) and a fluorescence imaging molecule without disrupting the overall structure of a platform protein (MjHsp). Fluorescently labelled LyP-Hsp exhibited specific interaction with macrophages, whereas macrophage-rich carotid lesions in mice showed good fluorescence signal after LyP-Hsp injection [26]. Therefore, demonstrated the potential of LyP-1- conjugated protein cages as a nano delivery of imaging agents for the diagnosis of atherosclerosis [26]. Qin et al., [27] formulated the gold nanorods conjugated with MMP2 antibody (AuNRs-Abs) for atherosclerosis mapping. After labelling with AuNRs-Abs, the distribution of MMP2 antibody of the atherosclerotic plaques was revealed using intravascular photoacoustic imaging (PAI) [27]. The data demonstrated that AuNRs-Abs is a promising probe for intravascular photoacoustic imaging (PAI), which quantitatively detect MMP2 antibody distribution [27]. 


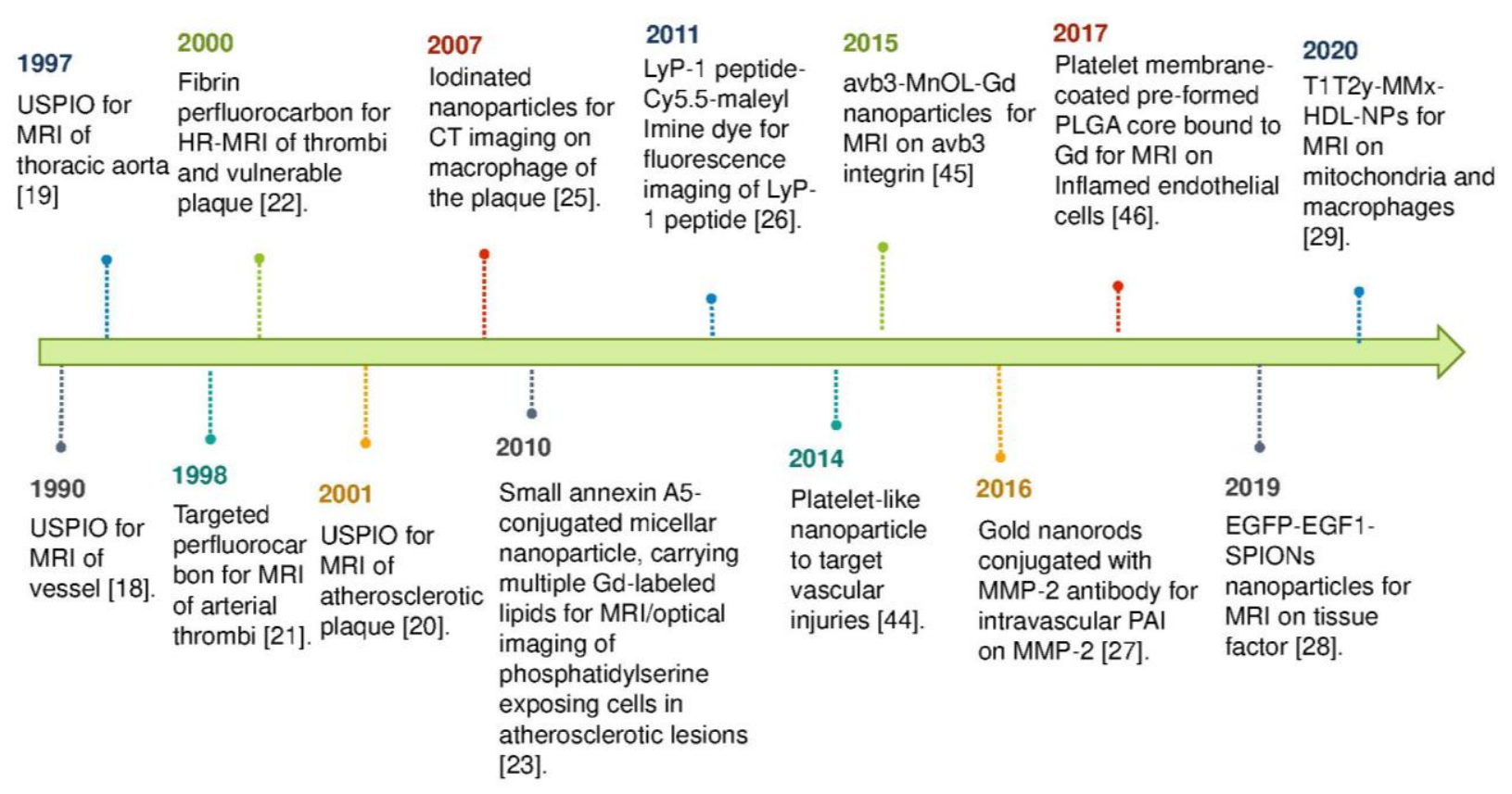

Fig. 1. Nanoparticles development for atherosclerosis imaging techniques

Recent investigations by Wei et al., [28] explored the conjugation of the 'fusion protein' enhanced green fluorescent protein with the first epidermal growth factor domain' (EGFP-EGF1) and superparamagnetic iron oxide nanoparticles (EGFP-EGF1- SPIONs) for molecular imaging of tissue factor (TF)-positive atherosclerotic plaques. It was observed that EGFP-EGF1-SPIONs provided an excellent contrast effects, thus improve the accuracy and great cytocompatibility in the detection of atherosclerotic plaques in vivo using MRI [28]. In short, EGFP-EGF1-SPION is a promising TF-targeting nanoprobe in detecting atherosclerotic plaques, which may improve the diagnosis of cardiovascular events for the comprehensive evaluation of atherosclerosis [28]. In more recent year, dual-targeted nanoparticles (NPs) with encapsulated iron oxide nanoparticle and magnetic resonance imaging (MRI) contrast agent were designated and synthesized by Banik et al., [29] to perform the double duty of diagnosis and therapy in atherosclerosis treatment regime. The optimized composition of the dual-targeted NP was found to reduce lipid levels in the body significantly without provoking any significant immunogenic effect inside the body [29]. This finding revealed the invention and optimization of a versatile NP platform, which can be further utilized as theranostic applications for the simultaneous diagnostic and therapy of atherosclerosis [29].

\section{Imaging Modalities in Atherosclerosis}

Accurate and reliable diagnosis of vulnerable atherosclerotic plaques before clinical manifestations is a prerequisite to identify high-risk patients and tailored therapy. In conjunction with present imaging advances that principally target to evaluate structural constituent of atherosclerosis, for instance; fibrous cap thickness and the size of the lipid core (Figure 2), the molecular and cellular imaging were also designed to visualize biological features of atherosclerotic plaques such as inflammation, angiogenesis, and matrix degradation in the living organism [28,29,30-37]. Although the rupture-prone plaque components are detectable by various imaging technologies, it remains challenging to differentiate between erosion-prone plaque and a stable plaque by imaging. This section review on the design and applications of different imaging platforms (Table 2), such as; computed tomography (CT), magnetic resonance imaging (MRI), positron emission tomography (PET). 


\section{Table 2}

Alternative 'target-specific' contrast agents used for atherosclerosis plaque characterizations in different types of imaging platforms

\begin{tabular}{|c|c|c|c|c|}
\hline Imaging & Nanoplatforms & $\begin{array}{l}\text { Targeted } \\
\text { molecule } \\
\end{array}$ & Outcomes & Year [Reference] \\
\hline \multirow[t]{4}{*}{ MRI } & $\begin{array}{l}\text { Peptide conjugated } \\
\text { amphiphilic molecules } \\
\text { containing gadolinium } \\
\text { (CREKA-DSPE-PEG2000- } \\
\text { DTPA-Gd) }\end{array}$ & Fibrin & $\begin{array}{l}\text { CREKA-DSPE-PEG2000-DTPA-Gd } \\
\text { exhibited positive fluorescence } \\
\text { signal of the fibrin, by increasing T1 } \\
\text { rate. }\end{array}$ & $2016[38]$ \\
\hline & $\begin{array}{l}\text { Gadolinium-bis-5- } \\
\text { hydroxytriptamide- } \\
\text { diethylenetriamine } \\
\text { pentaacetic acid (bis-5HT- } \\
\text { DTPA-Gd) }\end{array}$ & $\begin{array}{l}\text { Enzyme } \\
\text { myeloperoxidase } \\
\text { (MPO) }\end{array}$ & $\begin{array}{l}\text { Bis-5HT-DTPA-Gd demonstrated } \\
\text { sustained plaque enhancement on } \\
\text { T1-weighted }\left(\mathrm{T} 1_{\mathrm{w}}\right) \text { imaging, thus } \\
\text { emphasizing the potential for } \\
\text { atherosclerotic plaque detection }\end{array}$ & 2018 [39] \\
\hline & $\begin{array}{l}\text { Superparamagnetic iron- } \\
\text { oxide nanoparticles (SPIONs) }\end{array}$ & $\begin{array}{l}\text { Atheroma } \\
\text { plaque }\end{array}$ & $\begin{array}{l}\text { SPIONs showed reasonable signals } \\
\text { of the atheroma plaque, by } \\
\text { reducing } T 2 \text {-weighted ( } \mathrm{T} 2 \mathrm{w}) \\
\text { magnitude images, thus could be } \\
\text { interesting for development of } \\
\text { imaging tracer for atherosclerosis }\end{array}$ & $2017[40]$ \\
\hline & $\begin{array}{l}\text { Superparamagnetic iron- } \\
\text { oxide nanoparticles } \\
\text { conjugated with the fusion } \\
\text { protein (EGFP-EGF-SPIONs) }\end{array}$ & Tissue factor & $\begin{array}{l}\text { EGFP-EGF-SPIONs exhibited } \\
\text { accumulation in the plaque, by } \\
\text { resulting in plaque images } \\
\text { enhancement contrast of } T 2 w \text { MRI. }\end{array}$ & 2019 [28] \\
\hline PET & $\begin{array}{l}{ }^{89} \text { Zirconium conjugated } \\
\text { hyaluronan nanoparticles } \\
\left({ }^{89} \text { Zr-HA-NPs) }\right.\end{array}$ & Hyaluronan (HA) & $\begin{array}{l}{ }^{89} \mathrm{Zr} \text {-HA-NPs revealed PET signal } \\
\text { hotspot of the abdominal aorta, by } \\
\text { demonstrating high aortic PET } \\
\text { signal localized in macrophages- } \\
\text { rich areas. }\end{array}$ & $2017[41]$ \\
\hline $\mathrm{CT}$ & $\begin{array}{l}\text { 11- mercaptoundecanoic } \\
\text { acid coated gold } \\
\text { nanoparticles (11-MUDA- } \\
\text { AuNP) }\end{array}$ & Macrophage & $\begin{array}{l}\text { 11-MUDA-AuNP exhibited in } \\
\text { monocytes of the atherosclerotic } \\
\text { plaque, indicating the feasibility of } \\
\text { tracking labelled monocytes with } \\
\text { CT }\end{array}$ & 2016 [42] \\
\hline SPECT/ CT & $\begin{array}{l}\text { Technetium-99m radiation- } \\
\text { labelled natural H-ferritin } \\
\text { nanocage (99mTc-HFn) }\end{array}$ & $\begin{array}{l}\text { Transferrin } \\
\text { receptor } 1\end{array}$ & $\begin{array}{l}\text { 99mTc-HFn demonstrated intense } \\
\text { focal radiotracer uptake in the } \\
\text { aortas, for good localization and } \\
\text { detection of macrophage-rich } \\
\text { plaques }\end{array}$ & 2018 [43] \\
\hline
\end{tabular}

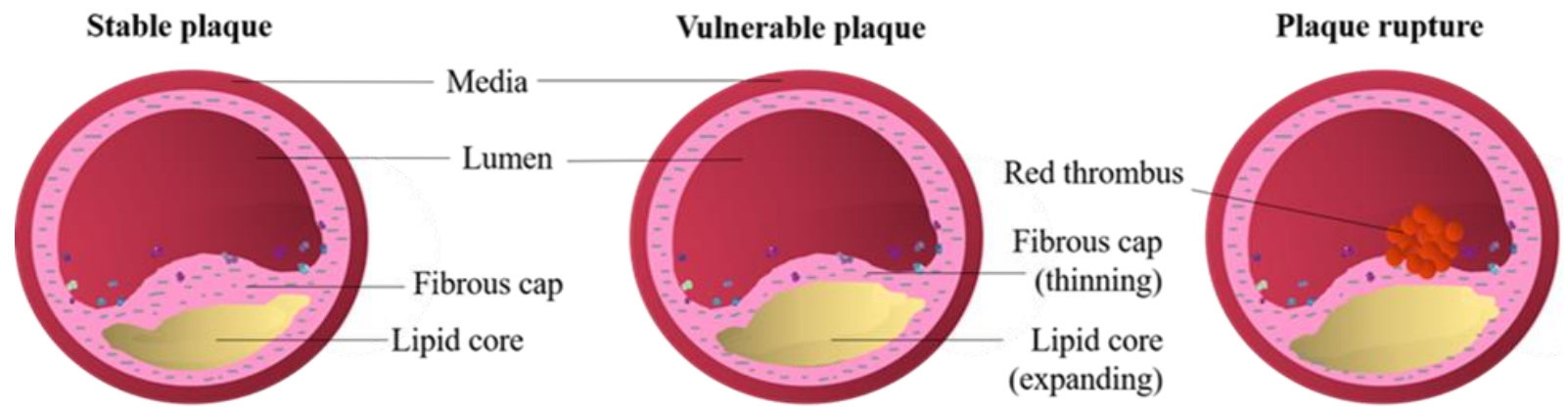

Fig. 2. Illustration of coronary thrombosis mechanism, where the formation of atherosclerosis plaques from stable to vulnerable was occurred. The thin fibrous caps are at risk of rupture 


\subsection{Computed Tomography (CT)}

Computed tomography (CT) is a convenient tool in diagnosing and evaluating the complexity of atherosclerosis throughout the entire vascular regions. CT may provide details on plaque configuration and abnormality, as well as the occurrence of vascular remodelling $[47,48]$. Its substantial impact on the diagnosis of subclinical atherosclerosis is in the identification and measurement of coronary calcium [49]. CT dominates an excellent temporal and spatial resolution, which can be further enhanced via multi-detector spiral computed tomography and the simultaneous utilization of iodinated contrast medium. This methodology allows the non-invasive detection of plaque locality and at the estimated magnitude [50]. The overall phenotypic category of plaques, together with the comparison among them, may also be acquired by intracoronary ultrasound [51]. Additionally, through engaging multi-colour (or spectral) CT, the composites incorporating tissues such as calcium deposits in atherosclerotic lesion can be analysed using different contrast vehicles, such as gold-labelled and iodine-labelled probe imaging. Thus, permitting instantaneous imaging of various atherosclerotic plaque mechanisms [52].

\subsection{Magnetic Resonance Imaging (MRI)}

Magnetic resonance imaging (MRI) offers incredibly accurate data on plaque magnitude, the occurrence of inflammation, and the state of the fibrous stratum [53,54]. MRI is possibly the most favourable approach for molecular imaging in atherosclerosis, because of the exceptional spatial (number of pixels utilized) and temporal (distance of time between the acquisitions of two images of the same area collected) resolution without demanding the management of ionizing radioactive nucleotides [55]. Additionally, MRI can also suggest the phenomenon of constructive vascular restoration, which holds the benefit of not utilize radiation exposure, thus providing an advantage to its usefulness in patient's convenient upon medical treatment [56].

To defeat the disadvantage of MRI that shows inadequate sensitivity during imaging, the application of paramagnetic vehicles such as gadolinium chelates and superparamagnetic iron oxide nanoparticles (SPIONs) contrast medium have been engaged to non-invasively image the arterial diseases [57]. In principle, these ultra-small paramagnetic iron oxide nanoparticles (USPIOs; 10-50nm in diameter) permit extravasation into vascular tissues allowing hypothetical assessment of atherosclerotic lesions [57]. This methodology has been applied for quantifying plaque macrophage load via MRI signal depletion as a result of phagocytic absorption of USPIOs in rabbits and in humans, where MRI signal concentrations associated with macrophage-rich unstable plaques $[20,58]$. However, mechanical problems correlated with vessel wall imaging using USPIOs indicates of low signal-to-noise ratio, reduced the specificity to visibly differentiate signal concerning anatomical sites, prolonged imaging periods and the complication in standardizing settings between scans [20].

In contrast, another research of USPIOs, involving the usage of peptides (VCAM-1) targeted nanoparticles for an in vivo study showed dissimilarity results to USPIOs. These nanoparticles were taken up by inflamed endothelium in accumulation to macrophages and vascular smooth muscle cells, permitting the discovery of fatty-streak lesions in MRI and optical imaging of juvenile hyperlipidemic Apo-lipoprotein E mice. Thus, enlightened the capability of this technique for primary intervention and monitoring of atherosclerosis [59].

Besides USPIOS, micro-particles consisted of iron oxide (MPIO) were suggested to have additional benefits in MRI imaging with their greater size $(0.9-8 \mu \mathrm{m})$. Unlike nano-particles iron oxide, which offered passive accumulation via macrophage uptake in atherosclerosis lesion, the larger particles size of MPIO has restricted them to localize at the endothelial surfaces that is suitable for 
endovascular molecular specificity [60]. The superior iron content brought by the MPIOs has also enhanced the signal recognition through high target-to-background ratio, thus allowed quick imaging of vascular inflammation with an exceptional level of specificity [60].

\subsection{Positron Emission Tomography (PET)}

Positron emission tomography (PET) is constructed based on the discovery of extraordinaryenergy photon pairs formed throughout annihilation impacts between positron and electron, after which 3D pictures are reassembled by computer analysis [61]. PET contrast agents comprising of positron-emitting radionuclides, such as fluorine-18 (18F), copper-64 (64Cu), and gallium-68 (68Ga) were manufactured from a cyclotron nearby the PET imaging capacity and chemically combined into the assembly of PET probes, thus applied instantly to prevent substantial decay with unlimited depth of penetration and excellent sensitivity $[61,62]$. Nevertheless, PET is a relatively expensive approach and has restricted accessibility because of the requirement of nearby cyclotron [62]. The main restriction of PET imaging is its low spatial resolution and shortage of anatomic reference frame [62]. The low spatial resolution can be improved by optical fluorescence imaging (OFI)/PET imaging, whereas additional CT or MRI imaging (PET/CT or PET/MRI) can overcome the limitation in anatomic reference frame [62].

\subsection{Single-Photon Emission Computed Tomography (SPECT)}

Single-photon emission computed tomography (SPECT) provides longer half-life of radionuclides than PET radionuclides, enabling the non-specific signal to be biologically washed out within the imaging time frame, leaving a cleaner signal of interest. Thus, makes SPECT imaging more practically available for imaging with longer radiosynthesis times and more affordable in price [63]. SPECT possessed comparable strengths and limitations with PET, which incorporate boundless depth penetration, great sensitivity to some extent, low spatial resolution and minimal 3D anatomical data [64]. Although SPECT is fundamentally less sensitive than PET, the shorter half-life of the PET radionuclides limits the accessibility of PET imaging thus, makes PET imaging more challenging [63].

Oxidized LDL-receptor-1 (LOX-1) is recognized as the primary receptor for oxidized LDL that has been associated with atherosclerosis-stimulating components and is considered to perform a central role in atherosclerosis [65]. An in vivo SPECT/CT imaging showed that the probe preferably bound to the region with vulnerable plaque features. Thus, this flexible multimodal imaging approach could be useful to identify characteristics of vulnerable plaque in high-risk atherosclerotic patients [66].

\subsection{Near Infrared Fluorescence (NIRF) Imaging}

Application of fluorescent imaging techniques in the NIR region has gained significant attention in the 21st century $[67,68]$. A satisfactory NIRF image requires excellent NIR coloration with superior image properties [69]. The ideal NIR imaging best work in the NIR spectrum $(600-900 \mathrm{~nm})$ due to a minimal interference of the fluorescence, a sensitive and non-invasive effect of imaging, and an improved tissue depth penetration [70,72]. The success of functionalized MRI-NIRF imaging was obtained by the conjugation of profilin- 1 antibodies with $\beta$-cyclodextrin magnetic nanoparticles (PFN1-CD-MNPs) loaded NIRF cyanine dye, that showed significantly high fluorescence signals in the aorta, leading to better atherosclerotic plaque targeted-imaging [73]. Another research on MRI-NIRF imaging of versatile ultra-small super-paramagnetic iron oxide (VUSPIO) similarly demonstrated 
excellent fluorescence signal in the plaque site, indicating potential of functional VUSPIO as a biocompatible and atheroma-specific diagnostic agent [85].

\subsubsection{Indocyanine Green (ICG)}

There are several NIRF dyes used for diagnosing and imaging atherosclerotic plaque known as cyanines. They are considered as preferable candidates due to improve physical properties and greatscale synthesis availability, whilst the most prominent of all is the indocyanine green (ICG) $[74,76-$ $78,86]$.

Iron oxide nanoparticles combined with indocyanine green (IONP-ICG) showed strong and intense fluorescence signals of arteriosclerotic plaques, thus considered to be the most effective dye for arteriosclerotic plaque visualization [79]. Likewise, ICG and the modified sirtuin 1 activator with a peptide targeting osteopontin (ICG / SRT@HSA-pept) exhibited strong fluorescence signals in the in vivo atherosclerotic lesions, allowing NIRF dye to be used clinically for cardiovascular disease nanotherapeutics [80]. Another interesting research was reported on the use of indocyanine green (ICG) loaded into a $90 \mathrm{~nm}$ contrast model, which developed using hemoglobin-depleted mice erythrocyteghosts known as near-infrared erythrocyte-derived transducers (NETs) [81]. NET was designed to specifically identify the locations of atherosclerotic lesions and occlusion, by detecting the fluorescence signal from NETs accumulation in inflammatory cells like macrophages. Using combined photoacoustic (PA) and fluorescence imaging tools, more findings to accurately distinguish symptomatic from asymptomatic coronary arteries in the future clinical detection could be achieved [81].

\subsubsection{Other cyanine-based dye}

Recent analysis on the development of extra domain B of fibronectin-specific nanoparticles (FNEDB-specific NPs) discovered cyanine accumulation in plaque-containing aortas, suggesting high NIRF intensity. This finding has suggested the possible opportunities for an improved diagnosis and therapy in high-risk atherosclerotic patients [82]. On the other hand, cyanine 5 (Cy5) monocytetargeting iron oxide magnetic nanoparticles (MNPs) demonstrated a possible targeting of NIRF dye for an early stage of atherosclerosis progression by exhibiting longer atherosclerosis plaque retention time in the aorta with substantial fluorescence signals [83].

In conclusion, NIRF is an effective imaging technique designed for envisioning both the high- and low-risk atherosclerotic plaque. Most of the NIRF studies have primarily exploited an FDA-approved NIRF dye of indocyanine green (ICG) to diagnose the unstable plaque permeability [84]. Clinical trials are required to properly evaluate the versatility of intravascular NIRF imaging using ICG along with several other novel NIRF dyes to better explain vulnerable plaque pathology, predict the outcomes and encourage customized pharmacotherapy of atherosclerotic plaques [84].

\section{Conclusions}

To date, advance research in diagnosing and assessing atherosclerosis are the outcome of integrated fields between cardiovascular medicine, immunology, medical imaging, bioengineering, and biochemical engineering. Although the application of the widespread imaging technique such as $M R I$ is yet to be recommended for routine assessment in the clinics, this non-invasive approach in characterizing atherosclerosis such as evaluating plaque structure and measuring inflammatory cells and progression is considered promising. The diagnostic supremacy of existing and evolving 
atherosclerosis imaging procedures will certainly encourage our strategy to patient care and assist determination towards cardiovascular study in establishment to greater comprehend disease systems and assess recent personalized medications. These objectives can be achieved throughout 1) better understanding of the complexity emphasizing the pathogenesis in atherosclerosis, 2) improve early detection of individual atherosclerosis risk and 3) assist in developing new targetedatherosclerosis therapies via the aids of imaging modalities including PET, SPECT and/or NIRF. Beside developing expertise and invention, additional research in patient-specific multimodal imaging approaches can also be personalized to disclose molecular signals with anatomic accuracy (such as assimilating information on plaque arrangement and local hemodynamic), cost-effectiveness and the consequence to patient's compliance.

\section{Acknowledgement}

The authors would like to extend their sincere appreciation to the Ministry of Education (MOE) Malaysia for funding this research through Fundamental Research Grant Scheme (FRGS/1/2018/STG07/UPM/02/4).

\section{References}

[1] World Health Organization. "World health statistics 2020: monitoring health for the SDGs, sustainable development goals." Geneva: World Health Organization (2020).

[2] Thomas, Hana, Jamie Diamond, Adrianna Vieco, Shaoli Chaudhuri, Eliezer Shinnar, Sara Cromer, Pablo Perel et al. "Global Atlas of Cardiovascular Disease 2000-2016: The Path to Prevention and Control." Global Heart 13, no. 3 (2018): 143-163. https://doi.org/10.1016/i.gheart.2018.09.511

[3] Grobbee, D. E. "Global Capacity Building for Global Challenges in Cardiovascular Disease." Global Heart 14, no. 4 (2019): 345-346. https://doi.org/10.1016/i.gheart.2019.10.005

[4] Basnet, Rajesh, Sandhya Khadka, Yuchun Wang, and Radheshyam Gupta. "Research Progress on Diagnosis and Treatment of Atherosclerosis." Science Letters 8, no. 1 (2020): 18-22.

[5] Zdrojewicz, Zygmunt, Mateusz Waracki, Bartosz Bugaj, Damian Pypno, and Krzysztof Cabała. "Medical applications of nanotechnology." Advances in Hygiene \& Experimental Medicine/Postepy Higieny i Medycyny Doswiadczalnej 69 (2015): 1196-1204. https://doi.org/10.5604/17322693.1177169

[6] Wang, Ji, Yu-Xia Kang, Wen Pan, Wan Lei, Bin Feng, and Xiao-Juan Wang. "Enhancement of anti-inflammatory activity of curcumin using phosphatidylserine-containing nanoparticles in cultured macrophages." International Journal of Molecular Sciences 17, no. 6 (2016): 969. https://doi.org/10.3390/ijms17060969

[7] The Royal Society \& The Royal Academy of Engineering. "Nanoscience and nanotechnologies: opportunities and uncertainties." The Royal Society. Clyvedon Press, Cardiff, UK (2004).

[8] Zia, Adil, Yuao Wu, Tuan Nguyen, Xiaowei Wang, Karlheinz Peter, and Hang T. Ta. "The choice of targets and ligands for site-specific delivery of nanomedicine to atherosclerosis." Cardiovascular research 116, no. 13 (2020): 20552068. https://doi.org/10.1093/cvr/cvaa047

[9] Jamalidinan, Fatemehsadat, Ali Fatehi Hassanabad, Christopher J. François, and Julio Garcia. "Four-dimensionalflow Magnetic Resonance Imaging of the Aortic Valve and Thoracic Aorta." Radiologic Clinics 58, no. 4 (2020): 753763. https://doi.org/10.1016/i.rcl.2020.02.008

[10] MacRitchie, Neil, Gianluca Grassia, Jonathan Noonan, Paul Garside, Duncan Graham, and Pasquale Maffia. "Molecular imaging of atherosclerosis: spotlight on Raman spectroscopy and surface-enhanced Raman scattering." Heart 104, no. 6 (2018): 460-467. https://doi.org/10.1136/heartjnl-2017-311447

[11] Chen, L., Z. Jiang, O. U. Akakuru, L. Yang, J. Li, S. Ma, and A. Wu. "Recent progress in the detection and treatment of atherosclerosis by nanoparticles." Materials Today Chemistry $17 \quad$ (2020): 100280. https://doi.org/10.1016/j.mtchem.2020.100280

[12] Schnitzler, Johan G., Kim E. Dzobo, Nick S. Nurmohamed, Erik SG Stroes, and Jeffrey Kroon. "Surmounting the endothelial barrier for delivery of drugs and imaging tracers." Atherosclerosis (2020): 1-9. https://doi.org/10.1016/i.atherosclerosis.2020.04.025

[13] Ou, Le-chun, Shan Zhong, Jing-song Ou, and Jin-wei Tian. "Application of targeted therapy strategies with nanomedicine delivery for atherosclerosis." Acta Pharmacologica Sinica (2020): 1-8. https://doi.org/10.1038/s41401-020-0436-0 
[14] Xu, Wan, Shuihua Zhang, Quan Zhou, and Wenli Chen. "VHPKQHR peptide modified magnetic mesoporous nanoparticles for MRI detection of atherosclerosis lesions." Artificial Cells, Nanomedicine, and Biotechnology 47, no. 1 (2019): 2440-2448. https://doi.org/10.1080/21691401.2019.1626411

[15] Pellico, Juan, Irene Fernández-Barahona, Marina Benito, Ángel Gaitán-Simón, Lucía Gutiérrez, Jesús Ruiz-Cabello, and Fernando Herranz. "Unambiguous detection of atherosclerosis using bioorthogonal nanomaterials." Nanomedicine: Nanotechnology, Biology and Medicine $17 \quad$ (2019): 26-35. https://doi.org/10.1016/i.nano.2018.12.015

[16] Wei, Qiuzhe, Jing Wang, Wei Shi, Bo Zhang, Huiwen Jiang, Mengyi Du, Heng Mei, and Yu Hu. "Improved in vivo detection of atherosclerotic plaques with a tissue factor-targeting magnetic nanoprobe." Acta Biomaterialia 90 (2019): 324-336. https://doi.org/10.1016/j.actbio.2019.04.014

[17] Sherin, Sainulabdeen, Sreedharan Balachandran, and Annie Abraham. "Curcumin incorporated titanium dioxide nanoparticles as MRI contrasting agent for early diagnosis of atherosclerosis-rat model." Veterinary and Animal Science (2020): 100090. https://doi.org/10.1016/i.vas.2020.100090

[18] Weissleder, Ralph, G. Elizondo, J. Wittenberg, C. A. Rabito, H. H. Bengele, and L. Josephson. "Ultrasmall superparamagnetic iron oxide: characterization of a new class of contrast agents for MR imaging." Radiology 175, no. 2 (1990): 489-493. https://doi.org/10.1148/radiology.175.2.2326474

[19] Anzai, Yoshimi, Martin R. Prince, Thomas L. Chenevert, Jeffrey H. Maki, Frank Londy Manette London, and Stuart J. McLachlan. "MR angiography with an ultrasmall superparamagnetic iron oxide blood pool agent." Journal of Magnetic Resonance Imaging 7, no. 1 (1997): 209-214. https://doi.org/10.1002/jmri.1880070132

[20] Ruehm, Stefan G., Claire Corot, Peter Vogt, Stefan Kolb, and Jörg F. Debatin. "Magnetic resonance imaging of atherosclerotic plaque with ultrasmall superparamagnetic particles of iron oxide in hyperlipidemic rabbits." Circulation 103, no. 3 (2001): 415-422. https://doi.org/10.1161/01.CIR.103.3.415

[21] Lanza, Gregory M., Christine H. Lorenz, Stefan E. Fischer, Michael J. Scott, William P. Cacheris, Robert J. Kaufmann, Patrick J. Gaffney, and Samuel A. Wickline. "Enhanced detection of thrombi with a novel fibrin-targeted magnetic resonance imaging agent." Academic Radiology 5 (1998): S173-S176. https://doi.org/10.1016/S10766332(98)80097-4

[22] Yu, Xin, Sheng-Kwei Song, Junjie Chen, Michael J. Scott, Ralph J. Fuhrhop, Christopher S. Hall, Patrick J. Gaffney, Samuel A. Wickline, and Gregory M. Lanza. "High-resolution MRI characterization of human thrombus using a novel fibrin-targeted paramagnetic nanoparticle contrast agent." Magnetic Resonance in Medicine: An Official Journal of the International Society for Magnetic Resonance in Medicine 44, no. 6 (2000): 867-872. https://doi.org/10.1002/1522-2594(200012)44:6<867::AID-MRM7>3.0.CO;2-P

[23] Van Tilborg, Geralda AF, Esad Vucic, Gustav J. Strijkers, David P. Cormode, Venkatesh Mani, Torjus Skajaa, Chris PM Reutelingsperger, Zahi A. Fayad, Willem JM Mulder, and Klaas Nicolay. "Annexin A5-functionalized bimodal nanoparticles for MRI and fluorescence imaging of atherosclerotic plaques." Bioconjugate Chemistry 21, no. 10 (2010): 1794-1803. https://doi.org/10.1021/bc100091q

[24] Wang, Kezheng, Dipanjan Pan, Anne H. Schmieder, Angana Senpan, Shelton D. Caruthers, Grace Cui, John S. Allen, Huiying Zhang, Baozhong Shen, and Gregory M. Lanza. "Atherosclerotic neovasculature MR imaging with mixed manganese-gadolinium nanocolloids in hyperlipidemic rabbits." Nanomedicine: Nanotechnology, Biology and Medicine 11, no. 3 (2015): 569-578. https://doi.org/10.1016/j.nano.2014.12.008

[25] Hyafil, Fabien, Jean-Christophe Cornily, Jonathan E. Feig, Ronald Gordon, Esad Vucic, Vardan Amirbekian, Edward A. Fisher, Valentin Fuster, Laurent J. Feldman, and Zahi A. Fayad. "Noninvasive detection of macrophages using a nanoparticulate contrast agent for computed tomography." Nature Medicine 13, no. 5 (2007): 636-641. https://doi.org/10.1038/nm1571

[26] Uchida, Masaki, Hisanori Kosuge, Masahiro Terashima, Deborah A. Willits, Lars O. Liepold, Mark J. Young, Michael V. McConnell, and Trevor Douglas. "Protein cage nanoparticles bearing the LyP-1 peptide for enhanced imaging of macrophage-rich vascular lesions." ACS Nano 5, no. 4 (2011): 2493-2502. https://doi.org/10.1021/nn102863y

[27] Qin, Huan, Yue Zhao, Jian Zhang, Xiao Pan, Sihua Yang, and Da Xing. "Inflammation-targeted gold nanorods for intravascular photoacoustic imaging detection of matrix metalloproteinase-2 (MMP2) in atherosclerotic plaques." Nanomedicine: Nanotechnology, Biology and Medicine 12, no. 7 (2016): 1765-1774. https://doi.org/10.1016/i.nano.2016.02.016

[28] Wei, Qiuzhe, Jing Wang, Wei Shi, Bo Zhang, Huiwen Jiang, Mengyi Du, Heng Mei, and Yu Hu. "Improved in vivo detection of atherosclerotic plaques with a tissue factor-targeting magnetic nanoprobe." Acta Biomaterialia 90 (2019): 324-336. https://doi.org/10.1016/j.actbio.2019.04.014

[29] Banik, Bhabatosh, Bapurao Surnar, Brett W. Askins, Mainak Banerjee, and Shanta Dhar. "Dual-Targeted Synthetic Nanoparticles for Cardiovascular Diseases." ACS Applied Materials \& Interfaces 12, no. 6 (2020): 6852-6862. https://doi.org/10.1021/acsami.9b19036 
[30] Murray, Christopher JL, and Alan D. Lopez. "Alternative projections of mortality and disability by cause 1990-2020: Global Burden of Disease Study." The Lancet 349, no. 9064 (1997): 1498-1504. https://doi.org/10.1016/S01406736(96)07492-2

[31] Tsimikas, Sotirios. "Noninvasive imaging of oxidized low-density lipoprotein in atherosclerotic plaques with tagged oxidation-specific antibodies." The American Journal of Cardiology 90, no. 10 (2002): L22-L27. https://doi.org/10.1016/S0002-9149(02)02958-2

[32] Wickline, Samuel A., and Gregory M. Lanza. "Nanotechnology for Molecular Imaging and Targeted Therapy." Circulation 107 (2003): 1092-1095. https://doi.org/10.1161/01.CIR.0000059651.17045.77

[33] Choudhury, Robin P., Valentin Fuster, and Zahi A. Fayad. "Molecular, cellular and functional imaging of atherothrombosis." Nature Reviews Drug Discovery 3, no. 11 (2004): 913-925. https://doi.org/10.1038/nrd1548

[34] Davies, John R., James H. Rudd, and Peter L. Weissberg. "Molecular and metabolic imaging of atherosclerosis." Journal of Nuclear Medicine 45, no. 11 (2004): 1898-1907.

[35] Lindner, Jonathan R. "Microbubbles in medical imaging: current applications and future directions." Nature Reviews Drug Discovery 3, no. 6 (2004): 527-533. https://doi.org/10.1038/nrd1417

[36] Strauss, H. William, Ravinder K. Grewal, and Neeta Pandit-Taskar. "Molecular imaging in nuclear cardiology." In Seminars in Nuclear Medicine, vol. 34, no. 1, pp. 47-55. WB Saunders, 2004. https://doi.org/10.1053/i.semnuclmed.2003.09.006

[37] Dobrucki, Lawrence W., and Albert J. Sinusas. "Cardiovascular molecular imaging." In Seminars in Nuclear Medicine, vol. 35, no. 1, pp. 73-81. WB Saunders, 2005. https://doi.org/10.1053/i.semnuclmed.2004.09.006

[38] Yoo, Sang Pil, Federico Pineda, John C. Barrett, Christopher Poon, Matthew Tirrell, and Eun Ji Chung. "Gadoliniumfunctionalized peptide amphiphile micelles for multimodal imaging of atherosclerotic lesions." ACS Omega 1, no. 5 (2016): 996-1003. https://doi.org/10.1021/acsomega.6b00210

[39] Rashid, Imran, Ghassan J. Maghzal, Yung-Chih Chen, David Cheng, Jihan Talib, Darren Newington, Minqin Ren et al. "Myeloperoxidase is a potential molecular imaging and therapeutic target for the identification and stabilization of high-risk atherosclerotic plaque." European Heart Journal 39, no. 35 (2018): 3301-3310. https://doi.org/10.1093/eurhearti/ehy419

[40] Prévot, Geoffrey, Tina Kauss, Cyril Lorenzato, Alexandra Gaubert, Mélusine Larivière, Julie Baillet, Jeanny LarocheTraineau et al. "Iron oxide core oil-in-water nanoemulsion as tracer for atherosclerosis MPI and MRI imaging." International Journal of Pharmaceutics 532, no. 2 (2017): 669-676. https://doi.org/10.1016/j.ijpharm.2017.09.010

[41] Beldman, Thijs J., Max L. Senders, Amr Alaarg, Carlos Pérez-Medina, Jun Tang, Yiming Zhao, Francois Fay et al. "Hyaluronan nanoparticles selectively target plaque-associated macrophages and improve plaque stability in atherosclerosis." ACS Nano 11, no. 6 (2017): 5785-5799.

[42] Chhour, Peter, Pratap C. Naha, Sean M. O'Neill, Harold I. Litt, Muredach P. Reilly, Victor A. Ferrari, and David P. Cormode. "Labeling monocytes with gold nanoparticles to track their recruitment in atherosclerosis with computed tomography." Biomaterials 87 (2016): 93-103. https://doi.org/10.1016/i.biomaterials.2016.02.009

[43] Liang, Minmin, Hui Tan, Jun Zhou, Tao Wang, Demin Duan, Kelong Fan, Jiuyang He et al. "Bioengineered H-ferritin nanocages for quantitative imaging of vulnerable plaques in atherosclerosis." ACS Nano 12, no. 9 (2018): 9300 9308. https://doi.org/10.1021/acsnano.8b04158

[44] Anselmo, Aaron C., Christa Lynn Modery-Pawlowski, Stefano Menegatti, Sunny Kumar, Douglas R. Vogus, Lewis L. Tian, Ming Chen, Todd M. Squires, Anirban Sen Gupta, and Samir Mitragotri. "Platelet-like nanoparticles: mimicking shape, flexibility, and surface biology of platelets to target vascular injuries." ACS Nano 8, no. 11 (2014): 1124311253. https://doi.org/10.1021/nn503732m

[45] Wang, Kezheng, Dipanjan Pan, Anne H. Schmieder, Angana Senpan, Shelton D. Caruthers, Grace Cui, John S. Allen, Huiying Zhang, Baozhong Shen, and Gregory M. Lanza. "Atherosclerotic neovasculature MR imaging with mixed manganese-gadolinium nanocolloids in hyperlipidemic rabbits." Nanomedicine: Nanotechnology, Biology and Medicine 11, no. 3 (2015): 569-578. https://doi.org/10.1016/j.nano.2014.12.008

[46] Wei, Xiaoli, Man Ying, Diana Dehaini, Yuanyuan Su, Ashley V. Kroll, Jiarong Zhou, Weiwei Gao, Ronnie H. Fang, Shu Chien, and Liangfang Zhang. "Nanoparticle functionalization with platelet membrane enables multifactored biological targeting and detection of atherosclerosis." ACS Nano 12, no. 1 (2018): 109-116. https://doi.org/10.1021/acsnano.7b07720

[47] Carrascosa, Patricia M., Carlos M. Capuñay, Pablo Garcia-Merletti, Jorge Carrascosa, and Mario J. Garcia. "Characterization of coronary atherosclerotic plaques by multidetector computed tomography." The American Journal of Cardiology 97, no. 5 (2006): 598-602. https://doi.org/10.1016/i.amjcard.2005.09.096

[48] Pohle, Karsten, Stephan Achenbach, Briain MacNeill, Dieter Ropers, Maros Ferencik, Fabian Moselewski, Udo Hoffmann, Thomas J. Brady, Ik-kyung Jang, and Werner G. Daniel. "Characterization of non-calcified coronary atherosclerotic plaque by multi-detector row CT: comparison to IVUS." Atherosclerosis 190, no. 1 (2007): 174-180. https://doi.org/10.1016/j.atherosclerosis.2006.01.013 
[49] Arad, Yadon, Kenneth J. Goodman, Marguerite Roth, David Newstein, and Alan D. Guerci. "Coronary calcification, coronary disease risk factors, C-reactive protein, and atherosclerotic cardiovascular disease events: the St. Francis Heart Study." Journal of the American College of Cardiology 46, no. 1 (2005): 158-165. https://doi.org/10.1016/i.jacc.2005.02.088

[50] Leber, Alexander W., Andreas Knez, Franz von Ziegler, Alexander Becker, Konstantin Nikolaou, Stephan Paul, Bernd Wintersperger et al. "Quantification of obstructive and nonobstructive coronary lesions by 64-slice computed tomography: a comparative study with quantitative coronary angiography and intravascular ultrasound." Journal of the American College of Cardiology 46, no. 1 (2005): 147-154. https://doi.org/10.1016/j.jacc.2005.03.071

[51] Leber, Alexander W., Andreas Knez, Alexander Becker, Christoph Becker, Franz von Ziegler, Konstantin Nikolaou, Carsten Rist et al. "Accuracy of multidetector spiral computed tomography in identifying and differentiating the composition of coronary atherosclerotic plaques: a comparative study with intracoronary ultrasound." Journal of the American College of Cardiology 43, no. 7 (2004): 1241-1247. https://doi.org/10.1016/i.jacc.2003.10.059

[52] Cormode, David P., Ewald Roessl, Axel Thran, Torjus Skajaa, Ronald E. Gordon, Jens-Peter Schlomka, Valentin Fuster et al. "Atherosclerotic plaque composition: analysis with multicolor CT and targeted gold nanoparticles." Radiology 256, no. 3 (2010): 774-782. https://doi.org/10.1148/radiol.10092473

[53] Choudhury, Robin P., Valentin Fuster, Juan J. Badimon, Edward A. Fisher, and Zahi A. Fayad. "MRI and characterization of atherosclerotic plaque: emerging applications and molecular imaging." Arteriosclerosis, $\begin{array}{lllllll}\text { Thrombosis, } \quad \text { and } & \text { (2002): } & \text { 1065-1074. }\end{array}$ https://doi.org/10.1161/01.ATV.0000019735.54479.2F

[54] Yuan, Chun, Shao-xiong Zhang, Nayak L. Polissar, Denise Echelard, Geraldo Ortiz, Joseph W. Davis, Elizabeth Ellington, Marina S. Ferguson, and Thomas S. Hatsukami. "Identification of fibrous cap rupture with magnetic resonance imaging is highly associated with recent transient ischemic attack or stroke." Circulation 105, no. 2 (2002): 181-185. https://doi.org/10.1161/hc0202.102121

[55] Xu, Wan, Shuihua Zhang, Quan Zhou, and Wenli Chen. "VHPKQHR peptide modified magnetic mesoporous nanoparticles for MRI detection of atherosclerosis lesions." Artificial Cells, Nanomedicine, and Biotechnology 47, no. 1 (2019): 2440-2448. https://doi.org/10.1080/21691401.2019.1626411

[56] Corti, Roberto, Zahi A. Fayad, Valentin Fuster, Stephen G. Worthley, Gerard Helft, James Chesebro, Michele Mercuri, and Juan J. Badimon. "Effects of lipid-lowering by simvastatin on human atherosclerotic lesions: a longitudinal study by high-resolution, noninvasive magnetic resonance imaging." Circulation 104, no. 3 (2001): 249 252. https://doi.org/10.1161/01.CIR.104.3.249

[57] Chen, Wei, David P. Cormode, Zahi A. Fayad, and Willem JM Mulder. "Nanoparticles as magnetic resonance imaging contrast agents for vascular and cardiac diseases." Wiley Interdisciplinary Reviews: Nanomedicine and Nanobiotechnology 3, no. 2 (2011): 146-161. https://doi.org/10.1002/wnan.114

[58] Kooi, Marianne Eline, V. C. Cappendijk, K. B. J. M. Cleutjens, A. G. H. Kessels, P. J. E. H. M. Kitslaar, M. Borgers, P. M. Frederik, M. J. A. P. Daemen, and J. M. A. Van Engelshoven. "Accumulation of ultrasmall superparamagnetic particles of iron oxide in human atherosclerotic plaques can be detected by in vivo magnetic resonance imaging." Circulation 107, no. 19 (2003): 2453-2458. https://doi.org/10.1161/01.CIR.0000068315.98705.CC

[59] Nahrendorf, Matthias, Farouc A. Jaffer, Kimberly A. Kelly, David E. Sosnovik, Elena Aikawa, Peter Libby, and Ralph Weissleder. "Noninvasive vascular cell adhesion molecule-1 imaging identifies inflammatory activation of cells in atherosclerosis." $\quad$ Circulation $114, \quad$ no. $14 \quad 1504-1511$. https://doi.org/10.1161/CIRCULATIONAHA.106.646380

[60] McAteer, Martina A., and Robin P. Choudhury. "Targeted molecular imaging of vascular inflammation in cardiovascular disease using nano-and micro-sized agents." Vascular Pharmacology 58, no. 1-2 (2013): 31-38. https://doi.org/10.1016/i.vph.2012.10.005

[61] Ametamey, Simon M., Michael Honer, and Pius August Schubiger. "Molecular imaging with PET." Chemical Reviews 108, no. 5 (2008): 1501-1516. https://doi.org/10.1021/cr0782426

[62] Zhao, Jianhong, Junwei Chen, Shengnan Ma, Qianqian Liu, Lixian Huang, Xiani Chen, Kaiyan Lou, and Wei Wang. "Recent developments in multimodality fluorescence imaging probes." Acta Pharmaceutica Sinica B 8, no. 3 (2018): 320-338. https://doi.org/10.1016/i.apsb.2018.03.010

[63] Pimlott, Sally L., and Andrew Sutherland. "Molecular tracers for the PET and SPECT imaging of disease." Chemical Society Reviews 40, no. 1 (2011): 149-162. https://doi.org/10.1039/B922628C

[64] Lu, Yu-Chen, Cheng-Xiong Yang, and Xiu-Ping Yan. "Radiopaque tantalum oxide coated persistent luminescent nanoparticles as multimodal probes for in vivo near-infrared luminescence and computed tomography bioimaging." Nanoscale 7, no. 42 (2015): 17929-17937. https://doi.org/10.1039/C5NR05623C

[65] Hu, Bo, Dayuan Li, Tatsuya Sawamura, and Jawahar L. Mehta. "Oxidized LDL through LOX-1 modulates LDL-receptor expression in human coronary artery endothelial cells." Biochemical and Biophysical Research Communications 307, no. 4 (2003): 1008-1012. https://doi.org/10.1016/S0006-291X(03)01295-6 
[66] Li, Dayuan, Amit R. Patel, Alexander L. Klibanov, Christopher M. Kramer, Mirta Ruiz, Bum-Yong Kang, Jawahar L. Mehta, George A. Beller, David K. Glover, and Craig H. Meyer. "Molecular imaging of atherosclerotic plaques targeted to oxidized LDL receptor LOX-1 by SPECT/CT and magnetic resonance." Circulation: Cardiovascular Imaging 3, no. 4 (2010): 464-472. https://doi.org/10.1161/CIRCIMAGING.109.896654

[67] Escobedo, Jorge O., Oleksandr Rusin, Soojin Lim, and Robert M. Strongin. "NIR dyes for bioimaging applications." Current Opinion in Chemical Biology 14, no. 1 (2010): 64-70. https://doi.org/10.1016/i.cbpa.2009.10.022

[68] Xu, Yuling, Mei Tian, Hong Zhang, Yuling Xiao, Xuechuan Hong, and Yao Sun. "Recent development on peptidebased probes for multifunctional biomedical imaging." Chinese Chemical Letters 29, no. 7 (2018): 1093-1097. https://doi.org/10.1016/i.cclet.2018.03.032

[69] Hilderbrand, Scott A., and Ralph Weissleder. "Near-infrared fluorescence: application to in vivo molecular imaging." Current Opinion in Chemical Biology 14, no. 1 (2010): 71-79. https://doi.org/10.1016/i.cbpa.2009.09.029

[70] Frangioni, John V. "In vivo near-infrared fluorescence imaging." Current Opinion in Chemical Biology 7, no. 5 (2003): 626-634. https://doi.org/10.1016/i.cbpa.2003.08.007

[71] Pansare, Vikram J., Shahram Hejazi, William J. Faenza, and Robert K. Prud'homme. "Review of long-wavelength optical and NIR imaging materials: contrast agents, fluorophores, and multifunctional nano carriers." Chemistry of Materials 24, no. 5 (2012): 812-827. https://doi.org/10.1021/cm2028367

[72] Dip, Fernando D., Mayank Roy, Steven Perrins, Rama Rao Ganga, Emanuele Lo Menzo, Samuel Szomstein, and Raul Rosenthal. "Technical description and feasibility of laparoscopic adrenal contouring using fluorescence imaging." Surgical Endoscopy 29, no. 3 (2015): 569-574. https://doi.org/10.1007/s00464-014-3699-z

[73] Zhang, Shuihua, Wan Xu, Peng Gao, Wenli Chen, and Quan Zhou. "Construction of dual nanomedicines for the imaging and alleviation of atherosclerosis." Artificial Cells, Nanomedicine, and Biotechnology 48, no. 1 (2020): 169179. https://doi.org/10.1080/21691401.2019.1699823

[74] Resch-Genger, Ute, Markus Grabolle, Sara Cavaliere-Jaricot, Roland Nitschke, and Thomas Nann. "Quantum dots versus organic dyes as fluorescent labels." Nature Methods 5, no. 9 (2008): 763-775. https://doi.org/10.1038/nmeth.1248

[75] Gonçalves, M. Sameiro T. "Fluorescent labeling of biomolecules with organic probes." Chemical Reviews 109, no. 1 (2009): 190-212. https://doi.org/10.1021/cr0783840

[76] Zhang, Shuihua, Wan Xu, Peng Gao, Wenli Chen, and Quan Zhou. "Construction of dual nanomedicines for the imaging and alleviation of atherosclerosis." Artificial Cells, Nanomedicine, and Biotechnology 48, no. 1 (2020): 169179. https://doi.org/10.1080/21691401.2019.1699823

[77] Cho, Eun Chul, Charles Glaus, Jingyi Chen, Michael J. Welch, and Younan Xia. "Inorganic nanoparticle-based contrast agents for molecular imaging." Trends in Molecular Medicine 16, no. 12 (2010): 561-573. https://doi.org/10.1016/j.molmed.2010.09.004

[78] Zheng, Cuifang, Mingbin Zheng, Ping Gong, Dongxue Jia, Pengfei Zhang, Bihua Shi, Zonghai Sheng, Yifan Ma, and Lintao Cai. "Indocyanine green-loaded biodegradable tumor targeting nanoprobes for in vitro and in vivo imaging." Biomaterials 33, no. 22 (2012): 5603-5609. https://doi.org/10.1016/j.biomaterials.2012.04.044

[79] Ikeda, Hiroyuki, Akira Ishii, Kohei Sano, Hideo Chihara, Daisuke Arai, Yu Abekura, Hidehisa Nishi, Masahiro Ono, Hideo Saji, and Susumu Miyamoto. "Activatable fluorescence imaging of macrophages in atherosclerotic plaques using iron oxide nanoparticles conjugated with indocyanine green." Atherosclerosis 275 (2018): 1-10. https://doi.org/10.1016/i.atherosclerosis.2018.05.028

[80] Ma, Sai, Seyed Mohammad Motevalli, Jiangwei Chen, Meng-Qi Xu, Yabin Wang, Jing Feng, Ya Qiu et al. "Precise theranostic nanomedicines for inhibiting vulnerable atherosclerotic plaque progression through regulation of vascular smooth muscle cell phenotype switching." Theranostics 8, no. 13 (2018): 3693-3706. https://doi.org/10.7150/thno.24364

[81] Liu, Yonggang, Taylor Hanley, Hao Chen, Steven R. Long, Sanjiv S. Gambhir, Zhen Cheng, Joseph C. Wu, Georges El Fakhri, Bahman Anvari, and Raiyan T. Zaman. "Non-Invasive Photoacoustic Imaging of In Vivo Mice with Erythrocyte Derived Optical Nanoparticles to Detect CAD/MI." Scientific Reports 10, no. 1 (2020): 1-9. https://doi.org/10.1038/s41598-020-62868-1

[82] Yu, Xin, Sheng-Kwei Song, Junjie Chen, Michael J. Scott, Ralph J. Fuhrhop, Christopher S. Hall, Patrick J. Gaffney, Samuel A. Wickline, and Gregory M. Lanza. "High-resolution MRI characterization of human thrombus using a novel fibrin-targeted paramagnetic nanoparticle contrast agent." Magnetic Resonance in Medicine: An Official Journal of the International Society for Magnetic Resonance in Medicine 44, no. 6 (2000): 867-872. https://doi.org/10.1002/1522-2594(200012)44:6<867::AID-MRM7>3.0.CO;2-P

[83] Gao, Cheng, Qiaoxian Huang, Conghui Liu, Cheryl HT Kwong, Ludan Yue, Jian-Bo Wan, Simon MY Lee, and Ruibing Wang. "Treatment of atherosclerosis by macrophage-biomimetic nanoparticles via targeted pharmacotherapy and sequestration of proinflammatory cytokines." Nature Communications 11, no. 1 (2020): 1-14. https://doi.org/10.1038/s41467-020-16439-7 
[84] Khraishah, Haitham, and Farouc A. Jaffer. "Intravascular molecular imaging to detect high-risk vulnerable plaques: current knowledge and future perspectives." Current Cardiovascular Imaging Reports 13, no. 2 (2020): 1-10. https://doi.org/10.1007/s12410-020-9527-z

[85] Larivière, Mélusine, Cyril Samuel Lorenzato, Laurent Adumeau, Samuel Bonnet, Audrey Hémadou, Marie-Josée Jacobin-Valat, Abdelmajid Noubhani et al. "Multimodal molecular imaging of atherosclerosis: Nanoparticles functionalized with scFv fragments of an anti- $\alpha$ llbß3 antibody." Nanomedicine: Nanotechnology, Biology and Medicine 22 (2019): 102082. https://doi.org/10.1016/i.nano.2019.102082

[86] Zhang, Juchen, Zengying Qiao, Peipei Yang, Jie Pan, Lei Wang, and Hao Wang. "Recent advances in near-infrared absorption nanomaterials as photoacoustic contrast agents for biomedical imaging." Chinese Journal of Chemistry 33, no. 1 (2015): 35-52. 